\title{
MSX Sources in the LMC: Dust-Enshrouded AGB Stars, Post-AGB Stars and PN
}

\author{
P.R. Wood \\ Research School of Astronomy \& Astrophysics, Australian National \\ University
}

M. Cohen

Radio Astronomy Laboratory, University of California, Berkeley

\section{Introduction}

The Midcourse Space (MSX) Experiment (Price 1995) detected large numbers of point sources in the LMC at $8.28 \mu \mathrm{m}$ (Egan et al. 2001). Near-infrared $J H K L$ imaging and monitoring of the 504 MSX sources in a region of size $3^{\circ} \times 3.5^{\circ}$ covering most of the LMC bar has been carried out. Spectra have been taken for most of the post-AGB stars and a selection of the AGB stars and red supergiants.

\section{Results}

Of the 504 MSX sources, 125 are red supergiants or foreground stars, 230 are dust-enshrouded AGB stars and 25 appear to be post-AGB stars (including three RCB stars). MACHO light curves exist for about half the AGB and post-AGB stars: all the AGB stars have been monitored in the infrared for about 3 years. The AGB stars visible in the MACHO data show long-period variability of a large amplitude but semi-regular nature. The maximum period observed for the common AGB stars is surprisingly small, about 500 days, although there are some stars with periods to 1000 days. The lack of longer periods may be due to the fact that MACHO was not able to see the redder object. The almostcomplete near-IR monitoring will test this suggestion. Three of the post-AGB star candidates have light curves indicating that they are $\mathrm{R}$ Coronae Borealis stars while others show secular luminosity changes. Spectra of the post-AGB star candidates show that about half these objects have $\mathrm{H} \alpha$ emission.

A preliminary report of these results can be found in Wood \& Cohen (2001) and a full paper is in preparation.

\section{References}

Egan, M.P., Van Dyk, S.D., Price, S.D. 2001, AJ, 122, 1844 Price, S.D. 1995, Space Sci.Rev., 74, 81

Wood P.R. \& Cohen, M. 2001, in Astrophysics and Space Science Library Vol. 265, Post-AGB Objects as a 